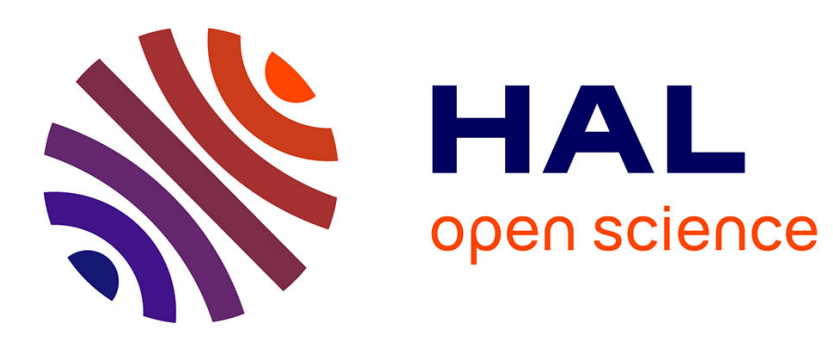

\title{
Chambre à étincelles démontable à feuilles minces
}

P. Savel, G. Ligonniére, T. Yuasa

\section{To cite this version:}

P. Savel, G. Ligonniére, T. Yuasa. Chambre à étincelles démontable à feuilles minces. Revue de Physique Appliquée, 1969, 4 (2), pp.311-312. 10.1051/rphysap:0196900402031100 . jpa-00243277

\section{HAL Id: jpa-00243277 https://hal.science/jpa-00243277}

Submitted on 1 Jan 1969

HAL is a multi-disciplinary open access archive for the deposit and dissemination of scientific research documents, whether they are published or not. The documents may come from teaching and research institutions in France or abroad, or from public or private research centers.
L'archive ouverte pluridisciplinaire HAL, est destinée au dépôt et à la diffusion de documents scientifiques de niveau recherche, publiés ou non, émanant des établissements d'enseignement et de recherche français ou étrangers, des laboratoires publics ou privés. 


\title{
GHAMBRE A ÉTINGELLES DÉMONTABLE A FEUILLES MINGES
}

\author{
P. SAVEL, G. LIGONNIÈRE et T. YUASA, \\ Institut de Physique Nucléaire, Facultés des Sciences de Paris et d'Orsay.
}

\begin{abstract}
Résumé. - Le besoin d'étudier certaines réactions nucléaires non coplanaires telles que $(\alpha, \alpha p)$ et $(\alpha, 2 \alpha)$ à moyennes énergies nous a incités à construire une chambre à étincelles démontable à feuilles d'Al de $0,06 \mathrm{~mm}$, commandée par l'accélérateur. Diverses caractéristiques de cette chambre ont été étudiées.
\end{abstract}

Abstract. - For studying some non coplanar nuclear reactions such as $(\alpha, \alpha p)$ and $(\alpha, 2 \alpha)$ at intermediate energy, we have constructed a spark chamber wich can be taken to pieces and which has thin Al foil electrodes. Its operation is controlled by the accelerator. Several characteristics have been studied.

I. La chambre. - Elle se compose de 30 cadres de plexiglas de dimensions intérieures : $300 \mathrm{~mm}$ $\times 300 \mathrm{~mm} \times 9 \mathrm{~mm}$. Chaque cadre comporte une gorge pour le joint torique de $\varnothing=2 \mathrm{~mm}$ qui assure l'étanchéité, une borne métallique assurant le contact électrique avec la feuille d'Al et accessible de l'extérieur, des trous pour l'évacuation et le remplissage de gaz et un petit trou permettant de vider et de remplir chaque intervalle entre les plaques. Ces cadres sont assemblés entre deux plaques d'Al portant une pression légèrement supérieure à la pression atmosphérique [1]. Cette chambre nous permet d'introduire ou de déplacer rapidement la cible ou éventuellement de changer certains éléments.

II. Système optique de prise de vues stéréoscopiques. - Le système optique permet de photographier deux vues à $90^{\circ}$, à l'aide de deux lentilles, d'un système de miroirs et d'une caméra ayant la distance focale de $65 \mathrm{~mm}$. Les conditions sont indiquées dans le tableau I.

III. Système électronique d'automatisation. - Il est constitué des parties suivantes : un ou plusieurs PM dont la porte s'ouvre par le tachymètre de l'accélérateur par intermédiaire d'un déphaseur, donnant un signal qui commande, après un retard variable, une série de triggers pour : application de la HT sur la chambre, éclairage des marques fiduciaires, marche d'un numérateur, avance film et simultanément fermeture d'une porte à temps variable qui interdit le passage de nouveaux signaux après le déclenchement de la chambre. Un générateur de porte à longue constante de temps $(1 \mu \mathrm{s} \leqslant t \leqslant 5 \mathrm{~s})$ est utilisé pour ouvrir cette porte. Un organigramme de l'utilisation de la chambre auprès du faisceau est présenté figure 1 .

IV. Déclenchement de la chambre. - En vue d'une utilisation de la chambre avec un faisceau du synchrocyclotron d'Orsay et devant travailler avec quelques paquets en structure fine dont l'intervalle est de $\sim 50 \mathrm{~ns}$ pour les protons et $\sim 90$ ns pour les $\alpha$, nous avons intérêt à minimiser les temps de retard et de montée de l'excitation de HT de la chambre. Nous avons comparé les thyratrons à hydrogène type $5 \mathrm{C} 22$ et $7620 \mathrm{HY}$ et un éclateur, et nous avons choisi le thyratron $5 \mathrm{C} 22$ qui donne la fréquence de répétition de $100 / \mathrm{s}$ pour $100 \%$ d'efficacité pour la HT de $7 \mathrm{kV}$.

TABLEAU I

\begin{tabular}{|c|c|c|c|c|}
\hline \multicolumn{3}{|c|}{ LENTILLES } & \multirow{2}{*}{$\begin{array}{c}\text { DistanGe } \\
\text { ENTRE LE CENTRE } \\
\text { DE LA CHAMBRE } \\
\text { ET GELUI } \\
\text { DE L'OBJEGTIF }\end{array}$} & \multirow[b]{2}{*}{$\begin{array}{c}\text { AGRANDISSEMENT } \\
\text { TOTAL }\end{array}$} \\
\hline Diamètre & $\begin{array}{c}\text { RAYONS } \\
\text { DE } \\
\text { GOURBURE }\end{array}$ & $\begin{array}{l}\text { Distance } \\
\text { FOGAle }\end{array}$ & & \\
\hline $\begin{array}{c}(\mathrm{cm}) \\
30\end{array}$ & $(\mathrm{~cm})$ & $(\mathrm{cm})$ & $(\mathrm{cm})$ & $10-9$ \\
\hline
\end{tabular}




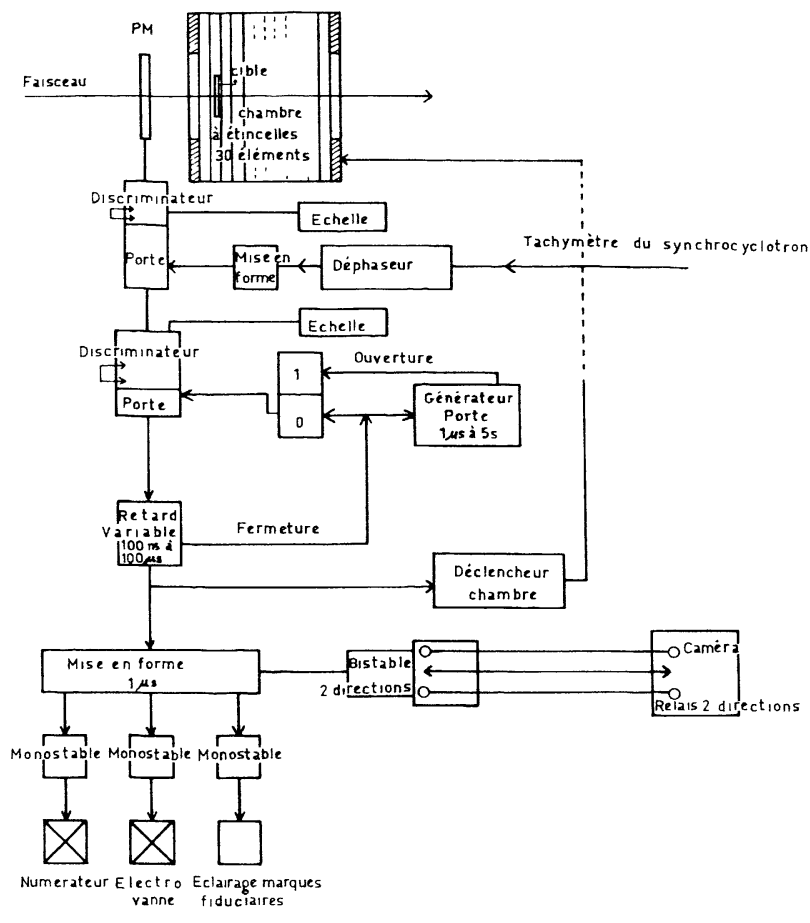

FIG. 1. - Organigramme du système électronique de la chambre utilisée avec le faisceau du synchrocyclotron.

V. Tension de balayage. - Nous avons étudié son influence sur le temps d'efficacité et sur le temps mort. D'après cette étude, nous avons fixé sa valeur à $50 \mathrm{~V}$, nous donnant ainsi une pleine efficacité pendant $\sim 300 \mathrm{~ns}$ et le temps mort de l'ordre de $1 \mu \mathrm{s}$.

VI. Efficacité de la chambre. - Il faut distinguer diverses sortes d'efficacités : efficacité de réponse de la chambre pour la traversée d'une particule chargée $\varepsilon_{\mathrm{ch}}$, celle de chacun des 30 éléments constituant la chambre $\varepsilon_{\mathrm{el}}$, celle pour l'angle d'inclinaison de la trajectoire par rapport au plan des électrodes $\varepsilon_{\theta}$ et celle de simultanéité de plusieurs trajectoires $\varepsilon_{\text {sim }}$. Ces temps dépendent de la HT, de la nature des gaz et aussi de la nature des particules. Avec nos meilleures conditions, et à l'aide d'une source $\beta$ de ${ }^{90} \mathrm{Y}$ simulant un faisceau et deux PM en coïncidence placés avant et après la chambre, nous avons mesuré ces efficacités : $\varepsilon_{\mathrm{ch}}$ et $\varepsilon_{\mathrm{e} 1} \geqslant 97 \%, \varepsilon_{\theta} \sim 80^{\circ}$. Quant à $\varepsilon_{\text {sim }}$, elle est très importante pour l'étude des réactions citées plus haut. Nous avons donc essayé de la déterminer avec deux sources $\beta$ de ${ }^{90} \mathrm{Y}$ et trois $\mathrm{PM}$ placés respectivement devant les sources et après la chambre et mis en coïnci- dence (deux fois de double coïncidence). Les résultats démontrent qu'on peut avoir la répartition de décharge identique pour deux trajectoires pour $\mathrm{HT}>8 \mathrm{kV}$

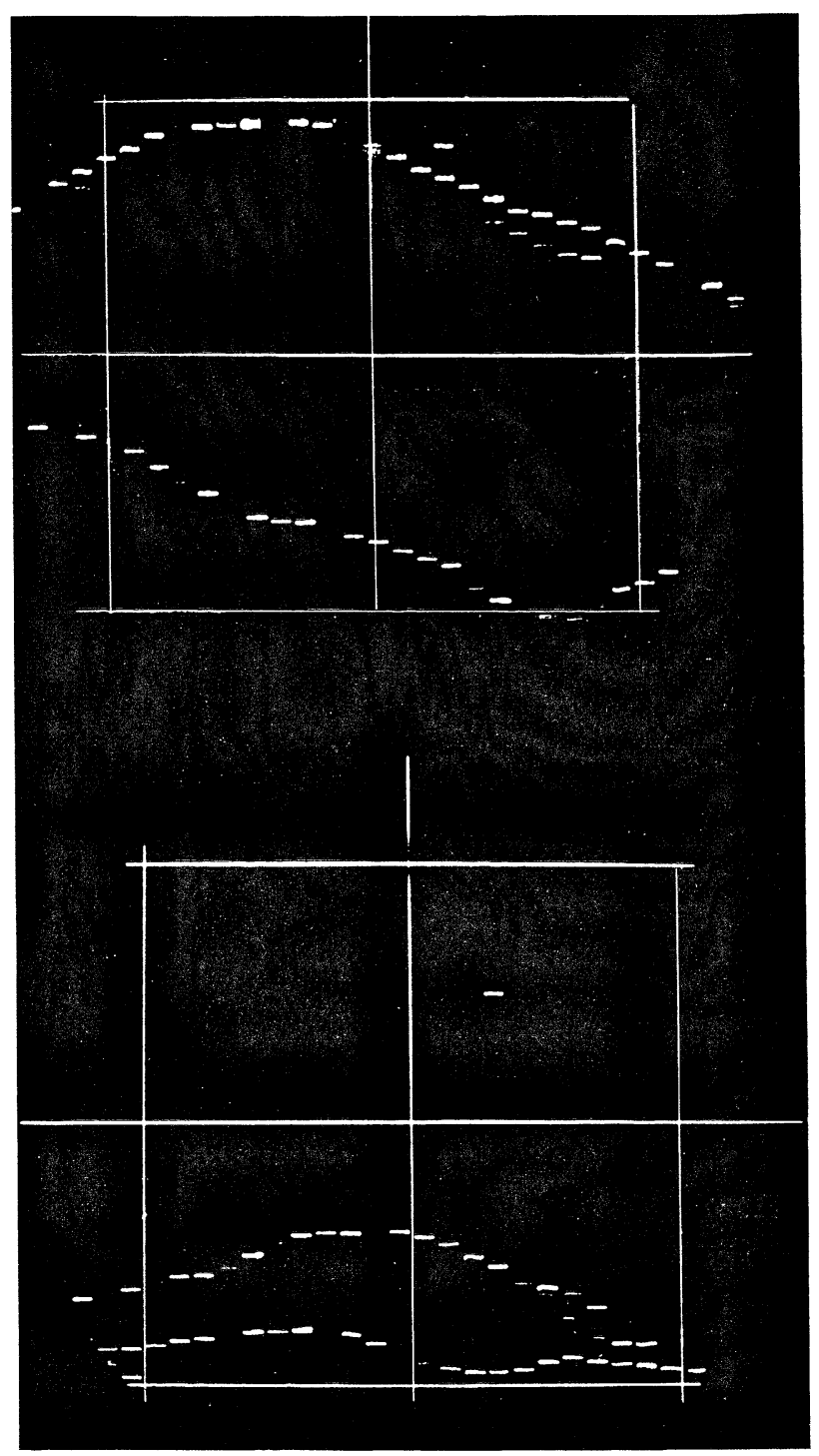

FIG. 2. - Vues stéréoscopiques à $90^{\circ}$ de deux trajectoires simultanées de rayons $\beta\left({ }^{90} \mathrm{Y}\right)$.

( fig. 2) et le temps de résolution de $10^{-7} \mathrm{~s}$. Deux très courts essais ont été faits avec les faisceaux de p et $\alpha$ produits par le synchrocyclotron d'Orsay. M. H. Itoh a participé à une partie de ce travail.

\section{BIBLIOGRAPHIE}

[1] Cette chambre a été inspirée par le travail de ANTOINE (P.), BAREYRE (P.), GAILlaRD (J. M.), RoBERT (G.) et SEIGE (R.), Nucl. Instr. Methods, 1963, 24, 418. 